From the Rocky Vista University College of Osteopathic Medicine in Ivins,

Financial Disclosures: None reported.

Support: This study was supported by an Intramural Research Grant from Rocky

Vista University. Siemens Medical Solutions loaned the ultrasonography scanner used in this study.

Address correspondence to Jing Gao, MD, Associate Professor and Director of Ultrasound, Rocky Vista University, 255 E Center St, Ivins, UT 84738-6790.

Email: jgao@rvu.edu Submitted May 20, 2019; revision received August 16, 2019; accepted September 30, 2019.

\title{
Ultrasound Shear Wave Elastography to Assess \\ Tissue Mechanical Properties in Somatic Dysfunction: A Feasibility Study
}

Jing Gao, MD; Judy Caldwell, DO; Michael Wells, PhD; David Park, DO

Context: Diagnosis of somatic dysfunction is based on subjective palpatory osteopathic assessments. This subjectivity has posed a challenge for researchers in studying osteopathic medicine. The development and use of radiological imaging techniques to objectively confirm or quantify muscle tissue stiffness associated with somatic dysfunction could be of benefit in osteopathic clinical practice, training, and further research.

Objectives: To investigate the feasibility of ultrasound shear wave elastography (SWE) to quantify muscle tissue stiffness in somatic dysfunction before and after osteopathic manipulative treatment (OMT).

Methods: In this prospective study, we assessed lumbar spine somatic dysfunction in 20 adult patients before and after a single OMT session using standard osteopathic palpatory assessments by osteopathic physician faculty members in the Department of Osteopathic Principle and Practice at Rocky Vista University College of Osteopathic Medicine (Utah campus). Shear wave velocity ( $\mathrm{SWV}, \mathrm{m} / \mathrm{s}$ ) was measured in lumbar paraspinal muscle tissue using a commercial ultrasonography scanner on all participants immediately before and after OMT. In this study, OMT techniques targeted the iliocostalis lumborum and included the articulatory technique, balanced ligamentous tension, facilitated positional release, high-velocity, low-amplitude technique, muscle energy, myofascial release, and the Still technique at the discretion of the osteopathic physician. The difference in SWV between muscle tissues with and without dysfunction, and differences in SWV of dysfunctional tissue before and after OMT were examined using unpaired and paired $t$ tests, as appropriate. The correlation between SWV measurements and osteopathic assessments was examined by the Spearman rank correlation. Intra- and interobserver reliability was analyzed using intraclass correlation coefficient.

Results: The difference in SWV between muscle tissues with and without somatic dysfunction was significant before OMT (mean [SD], 1.93 [0.44] vs 1.69 [0.19]; $P=.03$ ) and was not significant after OMT (mean [SD], 1.69 [0.19] vs 1.53 [0.31]; $P=.05$ ). The difference in SWV in the same tissue with somatic dysfunction before and after OMT was significant (mean [SD], $1.93[0.44]$ vs $1.52[0.3] ; P<.001$ ). The SWV value highly correlated with manual osteopathic assessments $(r=0.72)$. Intra- and interobserver reliability for performing SWE in somatic dysfunction was good (intraclass correlation coefficient $>0.80$ ).

Conclusions: The results of this study show that ultrasound SWE can objectively assess muscle tissue stiffness for diagnosis of somatic dysfunctions and for muscle tissue stiffness changes after OMT.

J Am Osteopath Assoc. 2020;120(10):677-684. Published online August 5, 2020. doi:10.7556/jaoa.2020.108

Keywords: OMT, osteopathic manipulative treatment, shear wave elastography, somatic dysfunction, ultrasound 
$\mathrm{T}$ he philosophic notion of body unity advocated by Andrew Taylor Still, MD, DO, was predicated on distinctive palpatory assessments performed by an osteopathic physician. ${ }^{1-4}$ Abnormal palpatory findings include tissue texture abnormality, asymmetry, restriction of motion, and tenderness (TART) ${ }^{5,6}$ Somatic dysfunction is diagnosed by the presence of any of the 4 TART criteria mentioned above and is defined as "impaired or altered function of related components of the somatic (body framework) system: skeletal, arthrodial, and myofascial structures, and related vascular, lymphatic, and neural elements." ${ }^{, 5} 6$ Somatic dysfunction has natural variability in clinical manifestations and almost always differs between patients. A challenge in osteopathic medicine is how to assess the location and severity of somatic dysfunction, as well as treatment effectiveness in an objective manner. $^{5}$

The therapeutic management of somatic dysfunction is traditionally accomplished through osteopathic manipulative treatment (OMT). ${ }^{1-4}$ OMT has been reported to have several clinical advantages, such as treating the patient's body and mind concurrently, high patient acceptance, and cost-effectiveness. ${ }^{7,8}$ As the osteopathic medical profession continues to grow, calls for more research are being heeded to provide evidence-based validation of OMT.

While conventional B-mode ultrasonography has been used to assess the morphology of skeletomuscular structures, it cannot provide information about tissue biomechanical properties (stiffness). Ultrasound shear wave elastography (SWE) is an emerging imaging technique that has been used to assess changes in tissue mechanical properties in liver fibrosis, tendinopathy, muscle physiologic conditions, and neuromuscular disorders. ${ }^{9-14}$ The principle of SWE is straightforward: acoustic radiation force impulse (ARFI) emitted from the ultrasound transducer pushes the tissue when the beam transmits to deep tissue. ARFI pulses cause tissue displacement perpendicular to the direction of the emission sound beam and produce shear waves (transverse waves). The speed of shear wave propaga- tion in the tissue depends on the stiffness of the tissue that the shear wave travels through. Shear wave propagation is fast in stiff tissue but slow in soft tissue. Using dedicated imaging processing software, the speed of shear wave propagation in the tissue is measured (shear wave velocity [SWV], meters per second). ${ }^{10-12}$ Since SWE can quantify tissue stiffness using computerized imaging processing, it may be considered a quantitative analog of the conventional palpatory examination.

SWE has a proven ability to assess muscle stiffness with variable causes. ${ }^{12-14}$ Hence, SWE may be useful in the evaluation of tissue stiffness associated with somatic dysfunction. Previously published conventional osteopathic methods for assessing somatic dysfunction $^{4-6}$ and the efficacy of OMT were insufficient in quality and quantity to affect practice changes. ${ }^{15,16}$ In an attempt to address these unmet needs in osteopathic medicine, we investigated the potential of SWE as a technique for quantifying somatic dysfunction.

\section{Methods}

This study was approved by the Rocky Vista University institutional review board. Twenty adult volunteer participants (10 men and 10 women; mean age, 28 years) were randomly recruited in the study. Participants, all of whom were students or employees of the university, provided written informed consent. Inclusion criteria required the study participants to be 20 years of age or older; to understand and sign informed consent; to be tolerant to osteopathic structural examination, ultrasonography scan, and OMT; to have no history of spinal surgery or trauma in the last 6 months; and to have no history of neuromuscular disorders, diabetic neuropathy, or autoimmune diseases.

Three osteopathic physicians (J.G., and 2 colleagues mentioned in Acknowledgements) from the Department of Osteopathic Principle and Practice, performed manual palpatory examinations of the lumbar region (L1-5) in 20 participants using standard protocol 
for assessing somatic dysfunction. Osteopathic assessments included evaluating skin changes (red reflex, skin drag, cold or hot temperature), muscle tone, paraspinal fullness, lumbar spine rotation, flexion, restricted motion, symmetry, and tenderness. The physician determined the location and parameters of osteopathic palpatory assessments for the diagnosis of somatic dysfunction before OMT, and then performed OMT. The same physician who conducted the initial osteopathic palpatory assessment to diagnose somatic dysfunction and performed the single OMT session, also performed the reassessment after OMT. OMT in this study was targeted to the iliocostalis lumborum, a muscle group commonly affected with low back pain conditions. OMT techniques used in the study included articulatory technique, balanced ligamentous tension (BLT), facilitated positional release (FPR), high-velocity, lowamplitude (HVLA), muscle energy, myofascial release, and the Still technique at the discretion of the osteopathic physician. A temporary skin marker was used to indicate the site of somatic dysfunction to ensure accurate subsequent SWV measurement in the same anatomic locations before OMT and after OMT.

SWE was then conducted on the paraspinal musculature of all the marked segments bilaterally using an Acuson S3000 ultrasonography system (Siemens Medical Solutions) equipped with 9L4 linear array transducer (4-9 MHz). Participants were lying in a prone position with back muscles relaxed (Figure 1A). $\mathrm{SWV}(\mathrm{m} / \mathrm{s})$ was measured in bilateral paraspinal musculature of the lumbar regions (L1-5; Figure 1B) across the segments indicated by the initial osteopathic diagnosis and markings. A single operator performed SWE on the same 10 participants 2 times to test intraobserver repeatability. Two different ultrasonography operators (J.G. and a medical student) performed SWE on the other 10 participants separately to test interobserver reproducibility.

Standard ultrasonography machine settings for B-mode (Figure 1C) and SWE (Figure 1D) of lumbar paraspinal muscle included scanning frequency of 7 $\mathrm{MHz}$, image depth of $4 \mathrm{~cm}$, tissue harmonic imaging, dynamic range of 65 , mechanical index of 1.6 , and the size of region of interest of $2.65 \mathrm{~cm} \times 1.0 \mathrm{~cm}$ (Figure 2). Shear wave propagation slows when the tissue attenuation increases with increased depth. As reported, artifactual changes in the SWE field begin at depths of approximately $4 \mathrm{~cm}$ using a scanning frequency of 7 to $9 \mathrm{MHz} .{ }^{17}$ Therefore, the image depth for paraspinal muscle SWE was standardized as $4 \mathrm{~cm}$ from the skin in this feasibility study. Each patient had 4 SWV measurements. The average of the 4 SWV measurements in each region of tissue was used for analysis.

SWV data of both normal and somatic dysfunction segments was compared with the osteopathic physicians' documented evaluation based on the osteopathic assessments before and after OMT. All data point variables were expressed as mean (SD). The mean SWV between the sites with and without somatic dysfunction was compared using an unpaired $t$ test. The difference in muscle SWV before and after OMT was evaluated using a paired $t$ test. Interobserver and intraobserver variation in performing SWE was tested using the intraclass correlation coefficient (ICC). The correlation between SWV and osteopathic assessment was analyzed by the Spearman rank correlation. A $P<.05$ was considered statistically significant. All statistical analyses were conducted by using SPSS software (version 25.4; IBM).

\section{Results}

Of 20 participants, $8(40 \%)$ had a history of low back pain ranging from 6 to 72 months prior to presentation, and the remaining 12 participants $(60 \%)$ did not have a history of low back pain. Mean body mass index of participants was $25 \mathrm{~kg} / \mathrm{m}^{2}$ (range, $21-32 \mathrm{~kg} / \mathrm{m}^{2}$ ). The mean depth of the subcutaneous tissue (the distance between the skin to paraspinal muscle) was $12 \mathrm{~mm}$ (range, 9-19 $\mathrm{mm}$ ). A significant difference was found in mean SWV between the sites with somatic dysfunction and the sites without somatic dysfunction (Figure 2) in the pre-OMT measurements ( $P=.03$; Table). No significant difference was found in mean SWV between the sites in post-OMT measurements $(P=.96)$. The difference in 

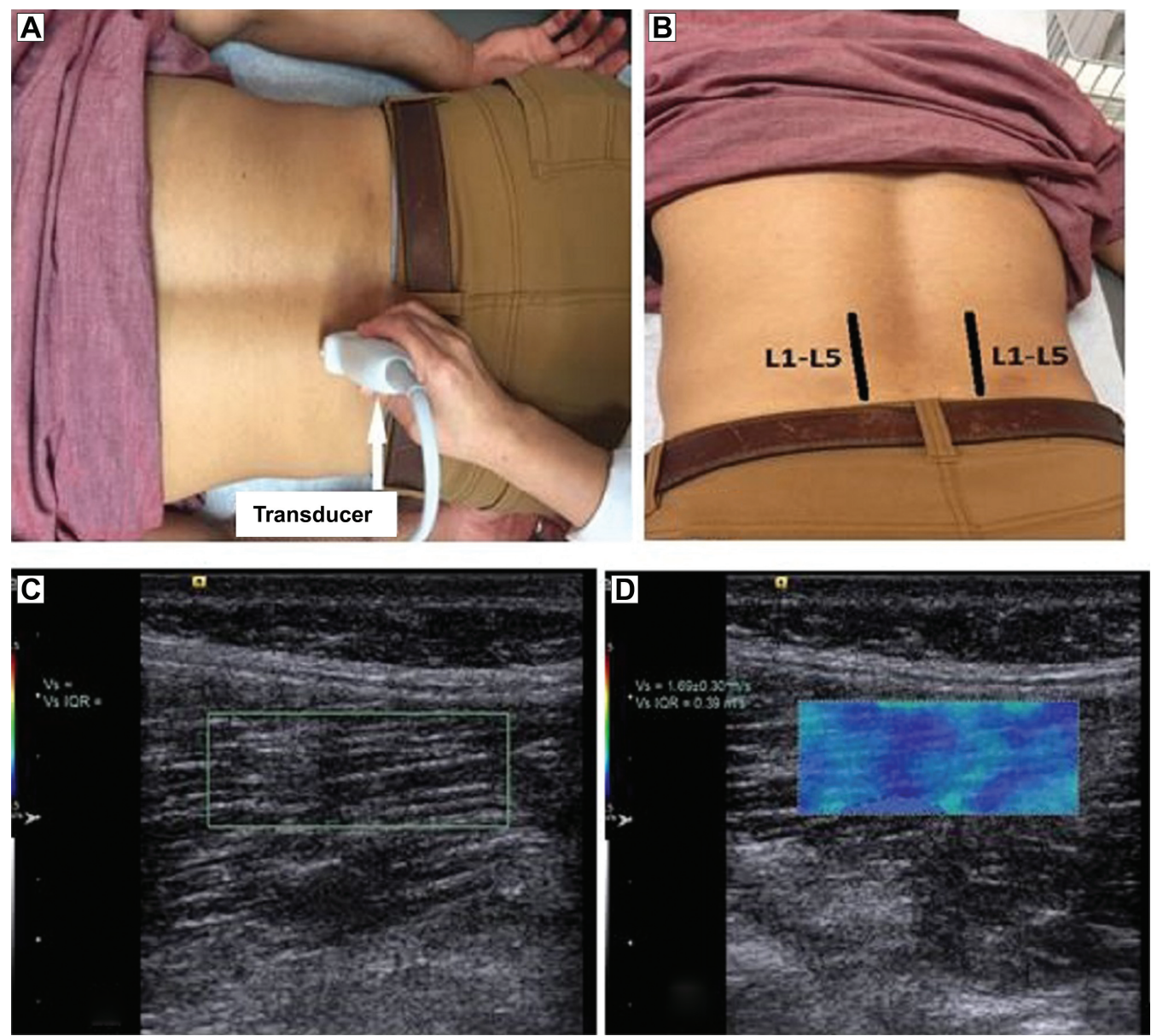

Figure 1.

Using a linear array ultrasound (US) transducer (A, white arrow), US shear wave elastography of bilateral paraspinal muscles (B, black vertical lines) was performed in the first and fifth lumbar vertebral (L1-L5) regions when the subject was in prone position with the back muscles relaxed. We used B-mode US image (C) and shear wave elastography (D) to reveal muscle tissue echotexture and to assess muscle tissue stiffness, respectively.

mean SWV in the region with somatic dysfunction before and after OMT (Figure 3) was also significant $(P<.001)$. The correlation of change in SWV to change in TART assessments was strong $(r=0.72)$. ICC for testing interobserver repeatability and intraobserver reproducibility in performing SWE was good (ICC, 0.80-0.97).

\section{Discussion}

This study demonstrates that SWE can document a significant difference in tissue mechanical properties (stiffness) between tissues with and without somatic dysfunction and in tissues with somatic dysfunctions before and after OMT. The concept of the using SWE to assess the tissue mechanical properties through measuring the speed of shear wave propagation in human tissue is well established. ${ }^{10-12}$ SWE has already been extended to various other clinical applications, including staging liver disease, distinguishing malignant vs benign thyroid nodules, and assessing pathologic changes of skeletal muscle in poststroke patients. ${ }^{17-19}$ Shear wave speed is estimated by using Virtual Touch IQ (Siemens Medical Solutions), a new 

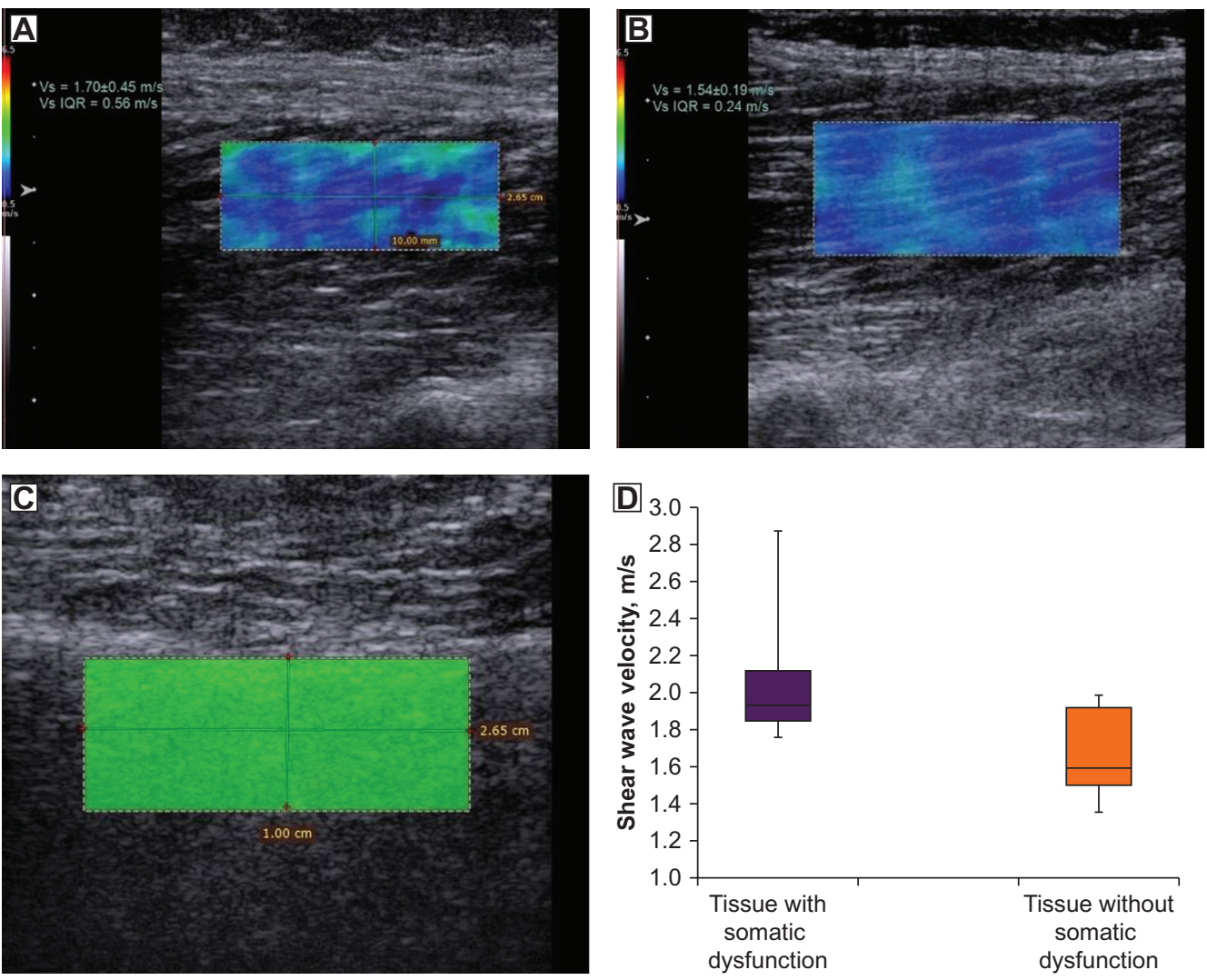

Figure 2.

Mean (SD) shear wave velocity of the tissue was measured (A) in the tissue with identified somatic dysfunction and (B) in the tissue free of identified somatic dysfunction. (C) Homogeneous green in the entire region of interest $(2.65 \mathrm{~cm} \times 1.0 \mathrm{~cm})$ in shear wave quality map indicates high quality of shear wave speed estimation and the shear wave velocity (SWV) measurement is valid for data analysis. (D) Box-and-whisker plots show that the difference in mean (SD) SWV (m/s) between the site with somatic dysfunction (purple box) and the site free of somatic dysfunction (orange box) before osteopathic manipulative treatment. The mean (SD) SWV was significantly higher in the tissue with somatic dysfunction than that without somatic dysfunction (1.93 [0.44] vs 1.69 [0.19], $P=.03)$. Abbreviation: SD, standard deviation.

ARFI-based reconstruction algorithm to calculate elasticity using the elastic modulus formula: $\mathrm{E}=3 \mathrm{pc}^{2}$ (E is the elastic modulus, $c$ is the shear wave speed, and $\mathrm{p}$ is tissue density). ${ }^{20} \mathrm{SWV}$, which measures the speed of shear wave propagation, is high in stiff and less elastic tissue but low in soft and more elastic

\section{Table.}

\section{Tissue Mean (SD) SWV Before and After OMT}

\begin{tabular}{llll} 
Parameter & Site with somatic dysfunction & Site free of somatic dysfunction & $\boldsymbol{P}^{*}$ value \\
\hline Mean (SD) SWV pre-OMT, m/s & $1.93(0.44)$ & $1.69(0.19)$ & .03 \\
\hline Mean (SD) SWV post-OMT, m/s & $1.52(0.3)$ & $1.53(0.31)$ & .96 \\
\hline$P$ value & $<.001$ & .05 & \\
\hline
\end{tabular}



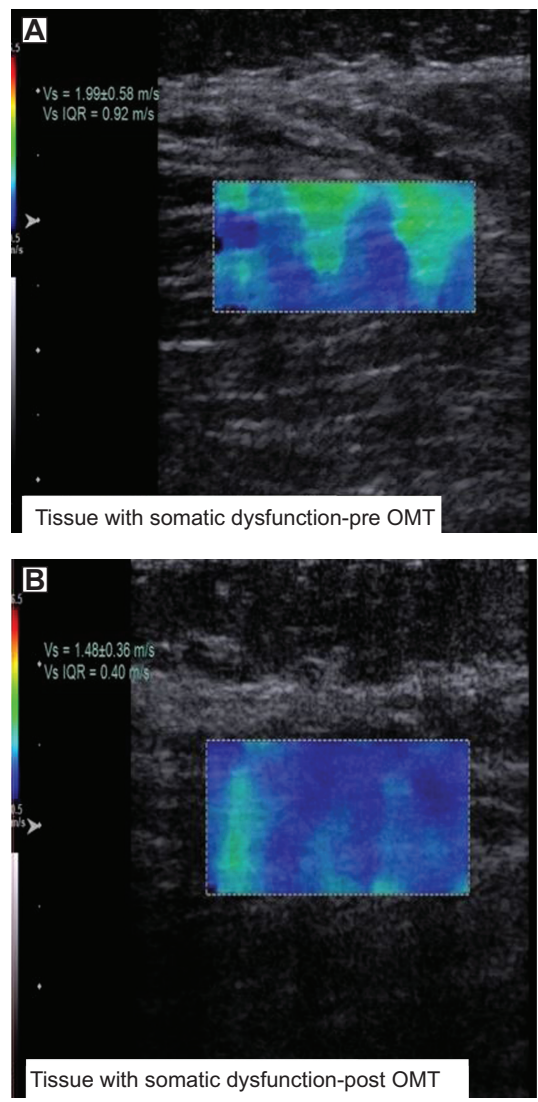

C

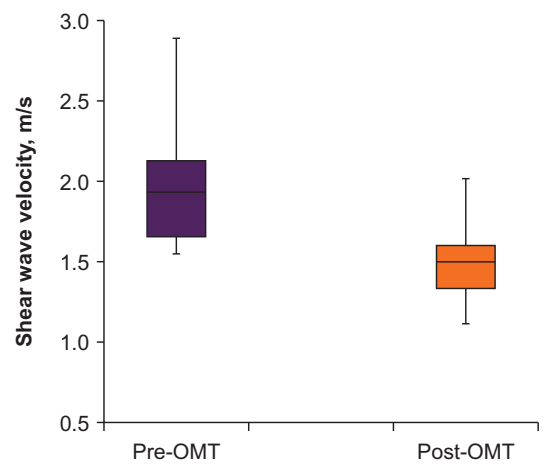

Figure 3.

$(A, B)$ The mean (SD) shear wave velocity (SWV, $\mathrm{m} / \mathrm{s}$ ) in the site with identified somatic dysfunction $(\mathrm{A})$ before and (B) after osteopathic manipulative treatment (OMT) is significantly different. SWV in the tissue with somatic dysfunction significantly decreased from $1.99(0.58) \mathrm{m} / \mathrm{s}$ to $1.48(0.36) \mathrm{m} / \mathrm{s}$ after OMT $(P<.001)$. (C) Box-and-whisker plots show that the difference in mean (SD) SWV in the tissue with somatic dysfunction before (purple box) and after (orange box) osteopathic manipulative treatment was significant (1.93 [0.44] vs $1.52[0.3], P<.001)$. tissue. Considering these properties, it is not surprising to notice a significant difference in mean SWV between the sites of soft tissue with somatic dysfunction and those without somatic dysfunction.

The SWE results of this study effectively demonstrate 2 things: first, that mean SWV can be used to quantify tissue property changes (stiffness and asymmetry) associated with somatic dysfunction, and second, that OMT can alter those tissue properties. The difference in mean SWV representing tissue stiffness between the sites with and without somatic dysfunction was significant $(P=.03)$. An increase of tissue stiffness in the region with somatic dysfunction may result from muscle contraction or spasm due to the pain, an imbalance of tissue activity, the development of muscle fibrosis, or combination of all of the above. Further, our data showed that the mean SWV in the region with somatic dysfunction before and after OMT was significantly different $(P<.05$; Table). The mean SWV in tissue in areas with somatic dysfunction significantly decreased post-OMT (Figure 3), suggesting quantitative documentation of the effectiveness of OMT techniques for improving soft tissue mechanical properties. $^{3-7}$ The ability of OMT to produce a change in the stiffness of tissue with somatic dysfunction may be related to the biomechanical effects of OMT on soft tissue. These include lengthening of myofascial, decreased muscle contractions, reduction of soft tissue restrictions, and increased flow and drainage of fluids. The various effects of OMT on neurologic control, autonomic nervous system functions, fascial contraction, muscular relaxation, and lymphatic circulation all potentially contribute to the change in mechanical properties of the local tissue after OMT. ${ }^{21,22}$

There were several limitations in this study. First, the sample size of 20 participants was small. Second, the correlation between pathology of the tissue with somatic dysfunction and the result of SWE is not available because definitive diagnosis through tissue biopsy is not the standard of care for patients with somatic dysfunction. Third, more than 1 osteopathic physician conducted the assessments and performed OMT on 
participants. However, each physician was assigned to 1 patient for continuity. Potential bias and variation in osteopathic assessments among these physicians could be significant, even if a standardized palpatory examination for assessing somatic dysfunction was used in the study. In addition, intra- and interobserver reliability of performing osteopathic palpatory assessments was not tested. Fourth, it was not possible to find participants who were completely free of any somatic dysfunctions to be classified as healthy controls. We randomly recruited 20 patients from the Rocky Vista University community (students and employees) in Ivins, Utah. Finally, somatic dysfunctions were treated with various OMT techniques individualized to each participant, which might have increased variation in the treatment effect in the study. However, this was done to emulate actual practices of osteopathic physicians, which also adheres to the osteopathic philosophy of treating each patient as an individual. Future studies on investigating the feasibility of SWE to assess the other somatic dysfunction characteristics of restriction of motion and tenderness, as well as the change in tissue after specific OMT techniques in a large population have been planned.

\section{Conclusion}

The results of this study strongly suggest that SWE is feasible to assess tissue property changes correlating to osteopathic somatic dysfunctions in adults by providing a quantitative measure of tissue stiffness. The results also suggest that SWE is useful in quantifying the change in soft tissue associated with tissue texture abnormalities after OMT. Findings further support the effectiveness of OMT techniques in the improvement of tissue properties in areas of somatic dysfunctions.

\section{Acknowledgements}

We thank Christopher Edwards, DO, Keith Bodrero, DO, and Keeling McLin, OMS II, for providing technical support for this study.

\section{Author Contributions}

All authors provided substantial contributions to conception and design, acquisition of data, or analysis and interpretation of data; all authors drafted the article or revised it critically for important intellectual content; all authors gave final approval of the version of the article to be published; and all authors agree to be accountable for all aspects of the work in ensuring that questions related to the accuracy or integrity of any part of the work are appropriately investigated and resolved.

\section{References}

1. Chila AG, executive editor. Section 1: Overview of the osteopathic medical profession. Foundation of Osteopathic Medicine. 3rd ed. Lippincott Williams and Wilkins. 2002.

2. Johnson SM, Kurtz ME. Conditions and diagnoses for which osteopathic primary care physicians and specialists use osteopathic manipulative treatment. J Am Osteopath Assoc. 2002;102(10):527-540.

3. Task Force on the Low Back Pain Clinical Practice Guidelines. American Osteopathic Association guidelines for osteopathic manipulative treatment (OMT) for patients with low back pain. J Am Osteopath Assoc. 2016;116(8):536-549. doi:10.7556/jaoa.2016.107

4. Burns DK, Wells MR. Gross range of motion in cervical spine: the effects of osteopathic muscle energy technique in asymptomatic patients. J Am Osteopath Assoc. 2006;106(3):137-142.

5. Licciardone JC, Kearns $\mathrm{CM}$. Somatic dysfunction and its association with chronic low back pain, back-specific functioning, and general health: results from the OSTEOPATHIC trial. J Am Osteopath Assoc. 2012;112(7):420-428

6. 2011 glossary of osteopathic terminology. American Association of College of Osteopathic Medicine website. www.aacom.org/docs/ default-source/insideome/got2011ed.pdf

7. Gamber R, Holland S, Russo D, Cruser DA, Hilsenrath PE. Cost-effective osteopathic manipulative medicine: a literature review of cost-effectiveness analysis for osteopathic manipulative treatment. J Am Osteopath Assoc. 2005;105(8):235-267.

8. Prinsenk JK, Hensel KL, Snow RJ. OMT associated with reduced analgesic prescribing and fewer missed work days in patients with low back pain: an observational study. J Am Osteopath Assoc. 2014;114 (2):90-98.

9. Rosskopf AB, Ehrmann C, Buck FM, Gerber C, Flück M, Pfirrmann CWA. Quantitative shear-wave US elastography of the supraspinatus muscle: reliability of the method and relation to tendon integrity and muscle quality. Radiology. 2015;278(2):465-474. doi:10.1148/ radiol.2015150908

10. Sande JA, Verjee S, Vinayak S, Amersi F, Ghesani M. Ultrasound shear wave elastography and liver fibrosis: a prospective multicenter study. World J Hepatol. 2017;9(1):38-47. doi:10.4254/wjh.v9.i1.38

11. Kot BCW, Zhang ZJ, Lee AWC, Leung VYF, Fu SN. Elastic modulus of muscle and tendon with shear wave ultrasound elastography: variations with different technical settings. PLOS ONE. 2012;7(8): e44348. doi:10.1371/journal.pone.0044348

12. Gennisson JL, Deffieux T, Mace E, Montaldo G, Fink M, Tanter M Viscoelastic and anisotropic mechanical properties of in vivo muscle tissue assessed by supersonic shear imaging. Ultrasound Med Biol. 2010;36(5):789-801. 
13. Chen J, O'Dell M, He W, Du LJ, Li PC, Gao J. Ultrasound shear wave elastography in the assessment of passive biceps brachii muscle stiffness: influence of sex and elbow position. Clin Imaging. 2017; 45(5):26-9.

14. Du LJ, He W, Cheng LG, Li S, Pan YS, Gao J. Ultrasound shear wave elastography in assessment of muscle stiffness in patients with Parkinson's disease: a primary observation. Clin Imaging 2016;40(6):1075-80

15. Steel A, Sundberg T, Reid R, et al. Osteopathic manipulative treatment: a systematic review and critical appraisal of comparative effectiveness and health economics research [published correction appears in Musculoskelet Sci Pract. 2017;30:86]. Musculoskelet Sci Pract. 2017;27:165-175. doi:10.1016/j.math.2016.10.067

16. Licciardone J, Gamber R, Cardarelli K. Patient satisfaction and clinical outcomes associated with osteopathic manipulative treatment. J Am Osteopathic Assoc. 2002;102(1):13-20.

17. Davis LC, Baumer TG, Bey MJ, van Holsbeek M. Clinical utilization of shear wave elastography in the musculoskeletal system. Ultrasonography. 2019;38:2-12
18. Cortez CD, Hermitte L, Ramain A, Mesmann C, Lefort T, Pialat JB. Ultrasound shear wave velocity in skeletal muscle: a reproducibility study. Diagn Interv Imaging. 2016;97(1):71-79. doi:10.1016/j. diii.2015.05.010

19. Gao J, Rubin JM, Chen J, O'Dell M. Ultrasound elastography to assess botulinum toxin $A$ treatment for post-stroke spasticity: a feasibility study. Ultrasound Med Biol. 2019;45(5):1094-1102. doi:10.1016/j.ultrasmedbio.2018.10.034

20. Sigrist RMS, Liau J, Kaffas AE, Chammas MC, Willmann JK. Ultrasound elastography: review of techniques and clinical applications. Theranostics. 2017;7(5):1303-1329. doi:10.7150/ thno. 18650 .

21. Vogel S. Evidence, theory and variability in osteopathic practice. Int J Osteopath Med. 2015;18:1-4.

22. Fryer G. Somatic dysfunction: an osteopathic conundrum. Int J Osteopath Med. 2016;22:52-63. doi:10.1016/j.josm.2016.02.002 ๑) 2020 American Osteopathic Association 